\title{
NEW METHOD FOR THE CORRECTION OF ENTROPION WITH TRICHIASIS BY TARSOTOMY*
}

\author{
BY \\ K. G. HADIJA \\ Eye Department, Civil Hospitals, Jinja, Uganda
}

FOR the correction of a shrunken conjunctiva and a misshapen tarsal plate by trachomatous scarring, many surgical methods can be used. The main aim is to correct the entropion and so eliminate the irritating trichiasis. Some of these methods are simple, others more complicated. The majority are plastic procedures designed to save tissue, but a few of them are more radical in nature. Some are concentrated on the lid margin, and others deal with the tarsal plate. Skin or oral mucous grafting may be used on the inter-marginal area of the lid, or the tarsal plate may be incised or totally removed. In areas where the incidence of trachoma is high and a large number of trachomatous cases demand surgical intervention, the most practical method is the simplest and quickest. Having in view that the modern treatment of trachoma with antibiotics combined with sulphonamides supports the choice of a method which is tissue-saving, I have found that the method described below fulfils these requirements, being a simple, quick, and tissue-saving procedure. The post-operative treatment is simple and does not require any special nursing attention.

\section{Method}

Anaesthesia.-The patient is lightly sedated pre-operatively. On the theatre table, both eyes are cleaned, drops of pantocain-adrenaline are instilled several times, and the lid margins are infiltrated with 2 per cent. Sol. Novutox. A dental syringe is useful for this purpose. In addition, I usually give a small amount of local anaesthetic to the outer canthus to eliminate spasm of the lower lid.

Surgical Procedure.-The Cruikshank lid everter is applied so that it clamps the lid skin about 1-2 mm. away from the eye-lashes with one branch, while the other plate-like branch lies just above the palpebral sulcus. The everter is then closed and fixed to rest on the patient's forehead; it can be stitched to the towels, or fixed in position by a clip. The lid is now ready for the incision, for the upper lid conjunctiva is well-exposed and the tarsal plate fully everted (Fig. 1a). The incision, with a Bard-Parker knife (Blade No. 15), runs about $1.5-2 \mathrm{~mm}$. from the lid margin through conjunctiva and the full thickness of the tarsal plate, starting in the region of the lacrimal punctum and ending in the outer corner of the lid. With scissors, the tarsal plate is then freed as far as the upper margin (Fig. 1b and c). In some cases, one finds the fatty tissue more developed, and this should be excised to make the lid thinner and lighter.

* Received for publication August 8, 1959. 


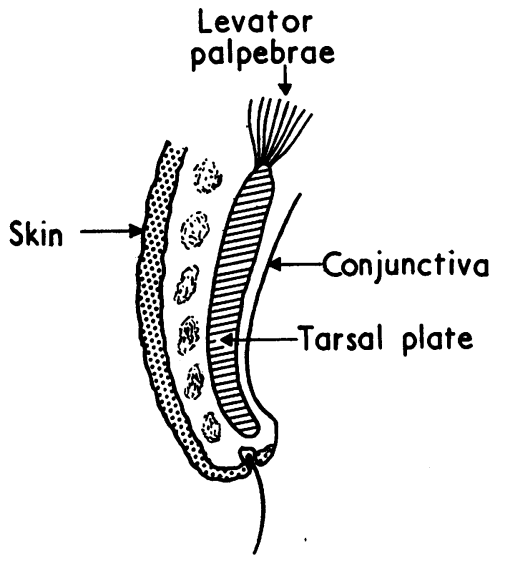

(a)

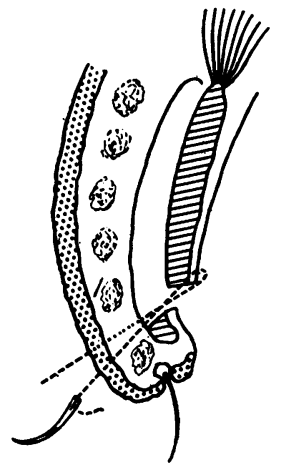

(d)

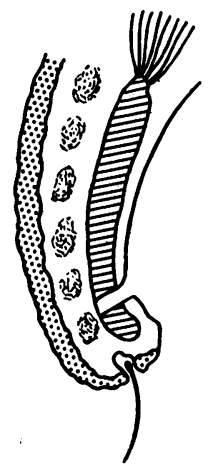

(b)

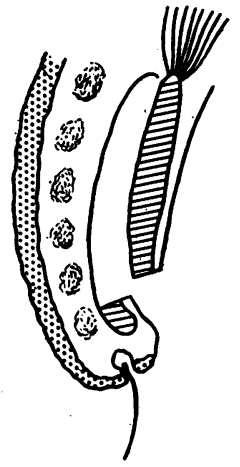

(c)

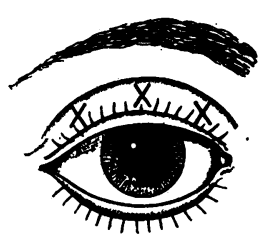

$(f)$

FIG. $1(a, b, c, d, e, f)$.-Steps in the operation, showing the mechanical principle of the method described.

The suturing material is a matter of choice. The stitching of the free tarsal margin can start from the skin or from the conjunctiva. When starting from the skin, a triangular eye-curved cutting needle, threaded with $2 / 0$ or $3 / 0$ catgut, passes just over the line of the eye-lashes through the lid, then through the conjunctiva near the free end of the tarsal plate, and back through the lid, coming out again just behind the line of eye-lashes (Fig. 1d). Three such sutures are applied, one in the outer, one in the middle, and one in the inner third of the lid.

If a start is made from the conjunctival surface, a double-threaded catgut suture is applied in the same way; both needles pass through the conjunctiva first, and are then tightened on the skin in the same positions.

When the sutures have been inserted, the lid everter is removed and the fold formed by the Cruikshank everter is stretched with a forceps by gently pulling the lid downwards. The effect can be seen at once on the operating table, as the lid margin is now slightly everted and the eye-lashes are directed forwards and up (Fig. 1e). The positions on the sutures are shown in Fig. 1(f). 
Achromycin ointment is then applied, both eyes are bandaged, and the patient is able to walk to the ward.

Post-operative Treatment.-This consists of daily dressing. The first dressing, after 24 hours, is carried out by cleaning the lids, applying oc. achromycin, and bandaging. The second dressing-48 hours after the operation-consists only of cleaning with an application of achromycin ointment twice a day, the eyes being left uncovered. This dressing is continued for a further 4 days; on the fifth day the stitches are removed, and on the sixth day the patient is discharged. For the whole of the time in hospital, the local application of antibiotics is combined with systemic treatment by sulphonamides.

Complications.-During the operation these are few and rare. Sometimes it is difficult to evert a very curved lid with a small and shrunken tarsal plate. In other cases, when the incision is made, there may be more bleeding than usual and ligation is necessary. A thin conjunctiva may be cut by the sutures, and in such cases it is safer to take some layers of tarsal plate into the stitch.

Patients are advised to continue the treatment with achromycin ointment twice a day for both eyes for a month, and then to report for a final examination.

Fig. 2 shows the pre-operative appearance and Fig. 3 the post-operative result in the same patient.

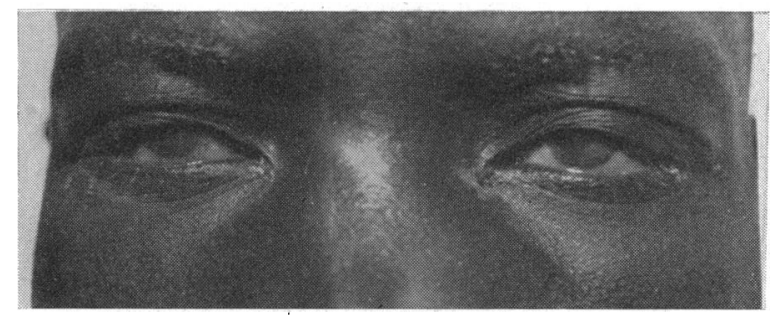

Fig. 2.-Pre-operative aspect.

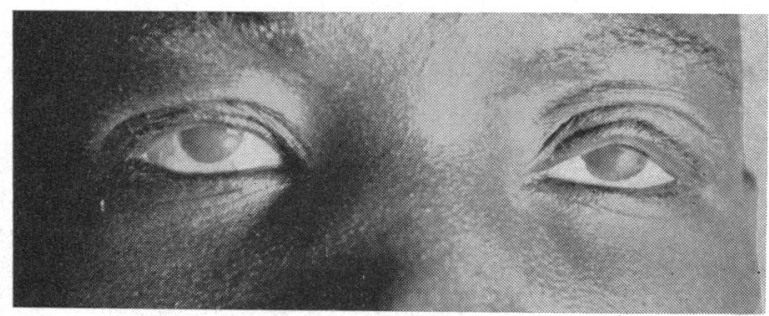

Fig. 3.-Post-operative result in the same patient.

\section{Discussion}

This method is easy, quick, and tissue-saving, and the post-operative treatment is simple, and recovery uneventful. The results obtained up to 
now are most satisfactory, and the cosmetic appearance good, restoring to the patient a normal appearance and not leaving the cornea exposed (Fig. 4).

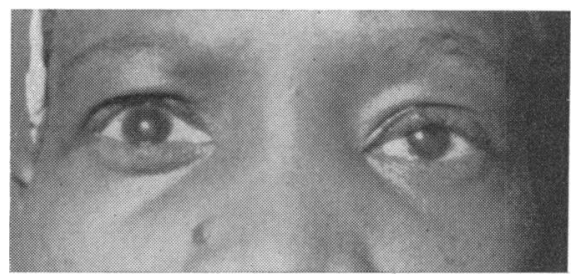

FIG. 4.-Right eye showing result of tarsectomy (eye-ball too exposed). Left eye showing result of tarsotomy by new method (eye almost normal in appearance).

The mechanical effect is achieved by cutting through the scarred area and stitching the larger portion of tarsal plate behind the smaller portion left on the lid margin, thus everting the lid margin with the eye-lashes. The effectiveness of this method is based on this mechanical principle.

Large numbers of patients have been operated on, and I have been able to follow-up most of them for 1 to 3 years since operation, and have found them free from trichiasis. The most probable cause of the few failures was that the sutures did not retain the tarsal plate in the correct position. To overcome this a small additional operation was necessary.

I should like to thank the Director of Medical Services, Uganda, for permission to publish, and Mr. E. F. King for his kindness in reading and commenting on this paper. 